\title{
Illumination for 360 Degree Cameras
}

\author{
I. Rakkolainen \\ University of Tampere \\ Communication Sciences \\ 33014 Tampere, Finland \\ csisra@sis.uta.fi
}

\author{
R. Raisamo \\ University of Tampere \\ Communication Sciences \\ 33014 Tampere, Finland \\ csrora@sis.uta.fi
}

\author{
M. Turk \\ University of California \\ Computer Science \\ Santa Barbara, CA 93106, USA \\ mturk@cs.ucsb.edu
}

\author{
T. Höllerer \\ University of California \\ Computer Science \\ Santa Barbara, CA, USA \\ holl@cs.ucsb.edu
}

\begin{abstract}
Additional illumination improves the capture of omnidirectional $360^{\circ}$ video and images, especially for dark or high-contrast environments. There is no "behind" for $360^{\circ}$ cameras, so the placement of lights is a problem. We explore ways to position lights on some $360^{\circ}$ cameras, and propose two good locations.
\end{abstract}

\section{CCS CONCEPTS}

- Computing methodologies $\rightarrow$ Mixed / augmented reality • Virtual reality $\bullet$ Hardware $\rightarrow$ Displays and imagers

\section{KEYWORDS}

Cinematic virtual reality; $360^{\circ}$ cameras; illumination; surround video; visualization; head-mounted display

\section{ACM Reference format:}

I. Rakkolainen, R. Raisamo, M. Turk, and T. Höllerer. 2018. Illumination for 360 Degree Cameras. In Proceedings of ACM VRST conference, Tokyo, Japan, November 2018 (VRST'18), 2 pages.

DOI: $10.1145 / 3281505.3281589$

\section{INTRODUCTION}

Omnidirectional $360^{\circ}$ cameras usually capture environments in daylight or in good illumination. Dark or high contrast spaces result in reduced image quality (grainy image, bad colors, poor contrast, etc.). Sometimes, no ambient light is available and additional illumination is needed, e.g., at night or in enclosed rooms.

In traditional filming, the lights are mostly behind the cameras. The $360^{\circ}$ cameras have no "behind," as they can directly see light panels around them, leading to unwanted bright spots and halos and the light panels partially blocking the view. Hidden lights behind objects in the scene limits the lighting and camera movement. Illumination for $360^{\circ}$ cameras is partly an unresolved problem. We present improved additional illumination for them.

Permission to make digital or hard copies of part or all of this work for personal or classroom use is granted without fee provided that copies are not made or distributed for profit or commercial advantage and that copies bear this notice and the full citation on the first page. Copyrights for third-party components of this work must be honored. For all other uses, contact the Owner/Author

VRST'18, November 28-December 1, Tokyo, Japan

(C) 2018 Copyright is held by the owner/author(s).

ACM ISBN 978-1-4503-6086-9/18/11.

https://doi.org/10.1145/3281505.3281589

${ }^{1}$ https://fb.com/ALLieCamera/ (Footnotes accessed on Sept. 29, 2018)

2 https://www.nctechimaging.com/istar/

${ }^{3}$ http://bounceimaging.com/

${ }^{4}$ http://www.serveball.com/

\section{RELATED WORK}

There are few previous works. Some infrared security and wildlife cameras use IR LEDs (outside the visible spectrum) around the lens. This method is employed (in black and white) by the ALLie ${ }^{1}$, iSTAR $^{2}$, Bounce Imaging ${ }^{3}$ and Squito $360^{\circ}$ cameras.

Computational cameras or 3D scanners may use patterned light to retrieve 3D geometry, e.g., Raskar et al. [1]. 3D laser scanner SpheronVR ${ }^{5}$ uses additional light to capture textures, but a scan of an environment may take even minutes.

Virtual cinematography [2] tries to relight the captured environment computationally, and augmented reality [3] adds synthetic photorealistic objects on top of reality, but these approaches do not help the $360^{\circ}$ video capture of dark environments.

\section{ILLUMINATION FOR $360^{\circ}$ CAMERAS}

We add illumination on surface or below some $360^{\circ}$ cameras (Samsung Gear 360, Nokia Ozo VR camera). We use thin strips of white, $120^{\circ}$ wide angle LEDs (pitch $17 \mathrm{~mm}$ ), which can be placed in tight spots and on free-form surfaces. We also built a setup with bright LED panels below a $360^{\circ}$ camera.
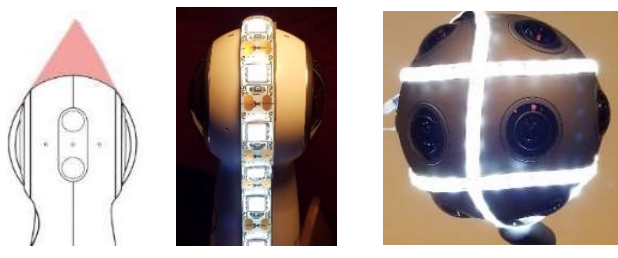

Figure 1: A cross-sectional view of the available volume for LED placement (in pink) around a Samsung Gear 360 camera top (left). An LED strip placed on the Gear 360 (middle). LEDs on the surface of a Nokia Ozo camera (right).

Placing the LEDs on a $360^{\circ}$ camera surface depends on the specific camera model. The lenses must not see any LEDs directly. Small cameras such as the Samsung Gear $360^{6}$ have little suitable surface area, but some volume above the surface is workable (see the pink volume on the upper part of Fig. 1 left). Larger cameras such as the Nokia Ozo ${ }^{7}$ VR camera have a larger surface area between the numerous lenses (Fig. 1 right). Also cameras such as Vuze ${ }^{8}$ or FITT $360^{9}$ enable more surface for lights.

\footnotetext{
${ }^{5}$ https://www.spheron.com/products/point-cloud-colourisation.html

${ }^{6} \mathrm{http}: / /$ www.samsung.com/global/galaxy/gear-360/

${ }^{7}$ https://ozo.nokia.com/

${ }^{8}$ https://vuze.camera/camera/vuze-plus-camera/

${ }^{9}$ http://fitt360.net/
} 
A camera usually can't see well below it and also the area is not usually important for the scene The area below has more space for LEDs than surface, thus creating a brighter image. Fig. 2 left shows an LED setup behind the Nokia Ozo camera. Fig. 2 middle shows a similar setup for the smaller Gear 360 . We also tested much brighter LED light panels (4x204 LEDs, total $1300 \mathrm{~lm}$ ) below the Gear 360 and a ring of 36 LEDs on top (Fig. 2 right).
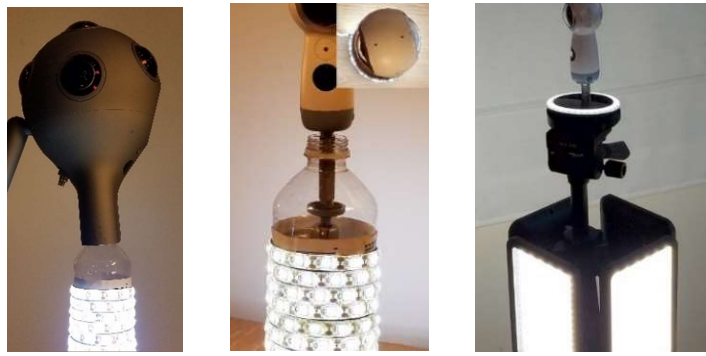

Figure 2: LEDs behind a Nokia Ozo camera (left). LEDs below the Samsung Gear 360 camera (middle), and the same seen from up (middle, insert). A bright LED panel light box below the Samsung Gear 360 camera (right).

\section{RESULTS}

Additional light improves the brightness and contrast in all ambient lighting, as can be seen in Figs. 3, 4, and 5. The best results are achieved in dark spaces. On the other hand, much of the light lands on the foreground, causing overexposure in near field.

Fig. 3 shows the difference between images taken without and with the LED strips on the Samsung Gear 360 camera in a dim room. The additional light is essential for the capture.
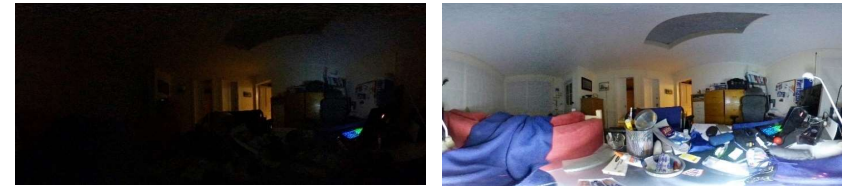

Figure 3: $360^{\circ}$ images without and with the LEDs in a dim lighting condition (LEDs on the surface of the Gear 360).

Fig. 4 shows the images taken without and with the LEDs in normal indoor lighting condition. The additional light improves the illumination especially on the foreground.
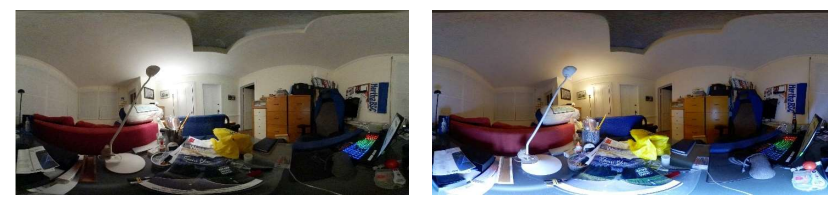

Figure 4: Images without and with the LEDs in normal indoor lighting condition (LEDs below the Gear 360).

An image of a room using the bright light box below the Gear 360 in daylight is depicted in Fig. 5. The light distribution is fairly even and uniform and the setup improves the capture.
The Samsung Gear 360 can accommodate 12 LEDs on its surface, whereas 120 LEDs can easily fit below it. The Nokia Ozo can have 120 LEDs on its surface, and even more behind or below it.
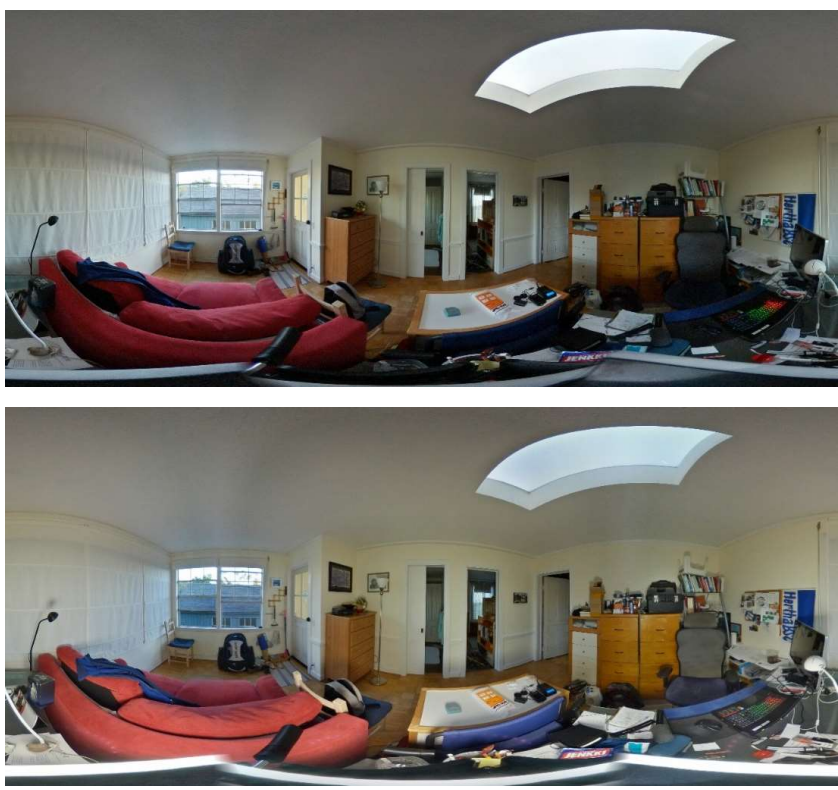

Figure 5: Images without and with the bright LED light box below the Gear 360 in a daylight indoor lighting condition.

\section{CONCLUSIONS}

We have presented methods to improve illumination for $360^{\circ}$ cameras. We attach the lights onto the camera surface or below it. This hides them from the view of the $360^{\circ}$ camera lenses and they can move along with the camera. The setup improves capture quality and is specifically important for dark or high-contrast environments. Our proof-of-concept prototype demonstrates the idea.

Placing the LEDs on the camera surface usually results in more uniform illumination, whereas LEDs below the camera can produce higher light output and longer illumination range.

In our future work we will analyze quantitative illumination data, the 3D structure of the environment, and address issues such as overexposure in near field, underexposure farther away, and unwanted shadows. A hybrid hardware/software solution with HDR, depth cameras and radiosity calculations can further improve $360^{\circ}$ imagery. Let there be light!

\section{ACKNOWLEDGMENTS}

We thank all the colleagues and reviewers who provided helpful comments on this paper. This work is partially funded by Business Finland (Tekes, \#1444/31/2016) and NSF grant \#IIS-1219261.

\section{REFERENCES}

[1] R. Raskar, K. Tan, R. Feris, J. Kobler, J. Yu, and M. Turk. 2005. Harnessing RealWorld Depth Edges with Multi-Flash Imaging. IEEE Computer Graphics and Applications 25 (Jan. 2005), 32-38.

[2] P. Debevec. 2006. Virtual Cinematography: Relighting through Computation. IEEE Computer 39 (Aug. 2006), 57-65.

[3] K. Agusanto, L. Li, Z. Chuangui, and N.W. Sing. 2003. Photorealistic Rendering for Augmented Reality Using Environment Illumination. In Proc. ISMAR 2003. IEEE, New York, NY, 208-216. 